\title{
Challenging scenarios in nontuberculous mycobacterial infection in cystic fibrosis
}

\author{
Stacey L. Martiniano ${ }^{1}$ () $\mid$ Charles R. Esther Jr. ${ }^{2}$ () $\mid$ Charles S. Haworth $^{3}$ | \\ Shannon H. Kasperbauer ${ }^{4,5}$ | Edith T. Zemanick ${ }^{1}{ }^{\circledR} \mid$ Lindsay J. Caverly $^{6}{ }^{(0)}$
}

${ }^{1}$ Department of Pediatrics, University of Colorado Anschutz Medical Campus, Aurora, Colorado

${ }^{2}$ Department of Pediatrics, University of North Carolina School of Medicine, Chapel Hill, North Carolina

${ }^{3}$ Cambridge Centre for Lung Infection, Royal Papworth Hospital NHS Foundation Trust,

Cambridge, UK

${ }^{4}$ Division of Mycobacterial and Respiratory Infections, National Jewish Health, Denver, Colorado

${ }^{5}$ Department of Medicine, University of Colorado, Denver, Colorado

${ }^{6}$ Department of Pediatrics, University of Michigan Medical School, Ann Arbor, Michigan

\section{Correspondence}

Lindsay J. Caverly, L2221 UH South, 1500

E. Medical Center Dr, Ann Arbor, MI 48109

Email: caverlyl@med.umich.edu

Funding information

Cystic Fibrosis Foundation,

Grant/Award Numbers: CAVERL17A0,

MARTIN17K0; National Heart, Lung, and

Blood Institute, Grant/Award Number:

K23HL136934

\begin{abstract}
This review summarizes the discussion of a session held during the 2018 North American Cystic Fibrosis (CF) Conference titled "Challenging Cases in Nontuberculous Mycobacterial (NTM) Management." In this session, a multidisciplinary panel of NTM experts discussed clinical challenges related to the management of NTM infection in people with CF in which decision-making falls outside of the Cystic Fibrosis Foundation/European Cystic Fibrosis Society NTM guidelines. Topics discussed included managing newly acquired NTM infection, selecting and monitoring treatment regimens, determining treatment endpoints, and caring for patients after NTM treatment.
\end{abstract}

\section{KEYWORDS}

cystic fibrosis, Mycobacterium abscessus complex, Mycobacterium avium complex, nontuberculous mycobacteria
Respiratory infections with nontuberculous mycobacteria (NTM) affect approximately $20 \%$ of people with cystic fibrosis (CF) and have increased in prevalence over the past two decades. ${ }^{1}$ NTM infections pose significant challenges for clinical management. The clinical course of NTM infection can be highly variable, ranging from transient, selfresolving infection to pulmonary disease associated with significant clinical decline, morbidity, and mortality. ${ }^{2}$ The nature of NTM treatment regimens further complicates decision-making. Recommended NTM treatment consists of at least one year of three or more antibiotics, with associated toxicities, costs, and burden of care. ${ }^{3}$

To address the clinical challenges of NTM infection in CF, the Cystic Fibrosis Foundation (CFF) and the European Cystic Fibrosis

Previously presented at the 2018 North American Cystic Fibrosis Conference.
Society (ECFS) jointly published guidelines on the management of NTM infection in CF in 2016. ${ }^{3}$ The CF specific guidelines are built on the general guidelines for NTM pulmonary disease published by the American Thoracic Society (ATS) and the Infectious Disease Society of America (IDSA) in 2007. ${ }^{4}$ The CFF/ECSF NTM guidelines provide a framework for the clinical approach to NTM infection in CF, including recommendations for NTM infection screening, clinical and microbiologic criteria for NTM pulmonary disease diagnosis, suggested treatment regimens, and monitoring schedules for drug toxicities.

At the North American CF Conference in 2018, a session titled "Challenging Cases in NTM Management" highlighted clinical scenarios in which decision-making falls outside of the published NTM guidelines. The session consisted of an interactive discussion between a multidisciplinary panel of NTM experts and the audience on approaches to these clinical situations based on available evidence and expert opinion. Challenges 
voiced by the audience and discussed with the panel included those related to managing of newly acquired NTM infection, selecting and monitoring treatment regimens, determining treatment endpoints, and caring for patients after NTM treatment.

In the following sections, we will review challenging scenarios of people with CF and NTM infection in which decision-making falls outside of the CFF/ECFS guidelines, and summarize the discussion of the expert panel and available literature.

\section{1 | MANAGEMENT OF NEWLY ACQUIRED NTM INFECTION}

\subsection{Should current medications be altered?}

At the time of initial NTM acquisition, one of the first considerations is whether the currently prescribed CF medications and treatments should be altered. For those on three times weekly azithromycin for immunomodulatory properties, discontinuing treatment is recommended once NTM is identified ${ }^{3}$ to avoid the development of NTM macrolide resistance. The panel recommended consideration of stopping chronic inhaled tobramycin as well, to minimize the development of NTM aminoglycoside resistance. Minimizing the use of other oral antibiotics with activity against NTM, including linezolid, minocycline, and moxifloxacin, should also be considered. However, the potential benefits of discontinuation of these antibiotics need to be weighed against their benefits in treating other CF pathogens, as optimization of treatment of non-NTM CF pathogens is a critical step in the determination of an NTM pulmonary disease diagnosis.

\subsection{How is NTM pulmonary disease diagnosed?}

Another primary consideration at the time of initial NTM acquisition is whether or not NTM pulmonary disease is present, as this will determine whether NTM treatment should be considered. The CFF/ECFS and ATS/ IDSA guidelines define NTM pulmonary disease broadly as signs and symptoms attributable to NTM infection, ${ }^{3,4}$ with microbiologic and clinical criteria that must be fulfilled for the diagnosis of NTM pulmonary disease. Certain clinical challenges arise in determining the fulfillment of each of these criteria. Microbiologic criteria for NTM pulmonary disease requires at least two positive cultures for the same NTM species from sputum and/or one positive bronchial wash or lavage (BAL) sample. ${ }^{3}$ This requirement can pose a challenge for patients that have difficulty expectorating sputum, and the alternative of bronchial wash or BAL typically requires general anesthesia in children. The panel discussed several approaches for obtaining acid-fast bacilli (AFB) cultures in nonexpectorating patients. The preferred option was induced sputum sampling, which is generally well-tolerated and has a high rate of success in obtaining an expectorated sputum sample. ${ }^{5,6}$ If NTM is suspected, but AFB cultures are negative, the panel recommended stopping antibiotics with activity against NTM for at least 2 weeks before the sample collection to increase the potential recovery of NTM. Finally, in the setting of Mycobacterium abscessus complex infection, the clinical laboratory may consider performing an AFB culture on an oropharyngeal (OP) swab sample. ${ }^{7}$ M. abscessus complex can be recovered from Burkholderia cepacia selective agar from OP swabs, and a positive culture with this method could contribute toward meeting microbiologic criteria for NTM pulmonary disease. Cultures of OP swabs, however, are less sensitive for M. abscessus complex than cultures of sputum or BAL samples, and are unlikely to detect Mycobacterium avium complex (MAC), so a negative OP swab culture is not sufficient to rule out NTM.

Determining the fulfillment of clinical criteria for NTM pulmonary disease poses additional challenges. A chest computed tomography (CT) is recommended at the time of a new NTM infection as part of the evaluation. Interpretation of the chest CT findings in regard to NTM pulmonary disease is often challenging, as many chest $\mathrm{CT}$ findings common in patients with CF (eg, bronchiectasis, tree-in-bud) are not specific for NTM infection, and can represent mucus plugging or other infections. While it is often not possible to distinguish between NTM and CF-related chest CT findings with certainty, nodules, tree-in-bud, cavitary lesions, and/or subsegmental atelectasis on chest $\mathrm{CT}$ raise the index of suspicion for NTM pulmonary disease, as does a progression of chest CT abnormalities if a comparator chest CT is available. ${ }^{8}$

\section{3 | Does CFTR modulator therapy impact treatment decisions?}

The panel discussed ways in which cystic fibrosis transmembrane conductance regulator (CFTR) modulator use may factor into the management of a newly acquired NTM infection. The impact that CFTR modulators will have on the prevalence and outcomes of NTM infection is unclear. If a patient with a new NTM infection has recently been started on a CFTR modulator, the panel recommended assessing the response to the CFTR modulator before diagnosing NTM pulmonary disease and initiating NTM treatment. In this situation, the initiation of a CFTR modulator can be considered a component of optimizing the CF treatment regimen in determining clinical criteria for NTM pulmonary disease. This recommendation may become more relevant with the recent approval of highly effective, triple modulator therapy. Finally, the diagnosis of NTM pulmonary disease is often subjective, and the decision to start NTM treatment is best made through a shared decision-making process with the physician and patients to weigh the risks and benefits of whether or not to initiate NTM treatment.

\section{2 | SELECTING AND MONITORING TREATMENT REGIMENS}

\subsection{What are the preferred treatment regimens?}

Once NTM pulmonary disease is diagnosed, the NTM treatment plan should be based on CFF/ECFS guidelines and chosen to target the species recovered in cultures, identified to the subspecies level for M. abscessus complex (M. abscessus subsp abscessus, M. abscessus subsp massiliense, $M$. 
abscessus subsp bolletii), and to the species level for MAC (eg, Mycobacterium avium, Mycobacterium intracellulare, Mycobacterium chimaera) (see guidelines for details of recommended treatment regimens). ${ }^{3}$ For patients with M. abscessus complex, skipping the IV intensive phase is not recommended. If a patient's respiratory cultures are persistently positive for both M. abscessus complex and MAC, treatment for both pathogens could be considered. In this scenario, the use of antibiotics such as azithromycin, clofazimine (if access via an Investigational New Drug [IND] is available), and amikacin would be optimal, as these antibiotics have activity against both M. abscessus complex and MAC. If multiple species or subspecies within the same NTM complex are persistently recovered from a patient's respiratory samples (eg, coinfection with both M. abscessus and Mycobacterium massiliense), the most drugresistant or difficult-to-treat pathogen should be targeted (eg, target $M$. abscessus, in this example).

\subsection{What about potential drug-drug interactions?}

NTM treatment should be adjusted for concomitant medications that may have drug-drug interactions. The most notable potential drugdrug interactions in NTM treatment in CF are between the rifamycins and the CFTR modulator ivacaftor. Rifampin strongly induces the metabolism of ivacaftor via cytochrome P450 making ivacaftor essentially inactive, and, therefore, should not be coadministered with CFTR modulators that contain ivacaftor. ${ }^{9}$ Rifabutin is a moderate cytochrome P450 inducer and should also be avoided in patients on ivacaftor if possible. For patients on CFTR modulators that contain ivacaftor, the panel discussed that the preferred alternative for rifamycins when treating MAC is clofazimine via an IND, or inhaled amikacin as a second alternative.

\subsection{What is the appropriate monitoring while on therapy?}

Establishing a clear monitoring plan for side effects and drug-related toxicities before starting treatment is key to reduce potentially toxicities. Equally important is specific counseling and education about drug toxicity and anticipated side effects with patients. Drug allergy or drug intolerances, such as nausea, are common and may emerge early in the course of treatment. Starting drugs in a staggered fashion (eg, several days or a week apart) can help to determine which drug may be the cause of a particular initial intolerance or reaction to allow intervention. In an effort to maximize efficacy and minimize renal toxicity, it is important to monitor drug levels when using intravenous aminoglycosides. Regular lab monitoring and clinical symptom assessment should occur and be tailored to the individual treatment plan. ${ }^{3}$ More specific monitoring plans discussed by the panel included recommendations for monthly hearing exams while on intravenous aminoglycosides, as well as baseline and interval electrocardiograms (EKGs) to monitor for a prolonged QT interval while on macrolides, clofazimine, and bedaquiline. Visual monitoring for color blindness while taking ethambutol and linezolid can be accomplished by patient self-assessment and by regular formal testing.

\subsection{How should drugs be adjusted if monitoring indicates a concern?}

Adjustments to treatment regimens may be indicated due to significant drug intolerances or side effects, drug-drug interactions, or lack of microbiologic and/or clinical response. The panel suggested using 6 months after treatment initiation as a useful time point to assess microbiologic and clinical response and determine if changes to the treatment plan are indicated. ${ }^{3}$ If a patient fails to convert to negative AFB cultures after 6 months of treatment, the panel discussed testing drug levels to ensure therapeutic dosing, ${ }^{10}$ checking or repeating drug sensitivity testing, and considering intensification of the NTM treatment regimen.

\section{3 | TREATMENT ENDPOINTS}

\subsection{When does NTM treatment end?}

The current standard of care in the management of NTM pulmonary disease is to continue therapy for 12 months beyond the date of conversion to negative cultures. ${ }^{3}$ If the clinical response has been satisfactory but treatment tolerance is an issue, the panel suggested that stopping NTM treatment after a minimum of 6 months of negative cultures could be considered. In an expectorating patient, cultures should be monitored monthly during NTM treatment. In a patient who is unable to expectorate sputum, home collection or sputum induction in the clinic should be considered. Bronchoscopy with a bronchial wash or BAL should also be considered in a patient who cannot produce sputum to ensure cultures have converted to negative. The panel discussed that the bronchial wash or BAL could be timed first after 3 to 6 months of treatment to test for conversion to negative culture, and again at the end of the treatment course (ie, 12 months after culture conversion). Although less sensitive than AFB culture of sputum or BAL fluid, AFB culture of OP swab samples can also be considered for monitoring response to treatment for M. abscessus complex. ${ }^{7}$ However, given the lower sensitivity of OP swab AFB cultures, negative cultures need to be confirmed by induced sputum or BAL. Some centers may elect to rely on clinical and/or radiographic outcomes in the setting where cultures cannot be collected routinely (ie, patients who cannot expectorate sputum). The panel discussed following a highresolution chest CT every 6 to 12 months during NTM treatment, including at the beginning and end of treatment. In the setting of cavitary disease, chest radiographs may suffice when monitoring for the closure of the cavity. Also discussed was the option to use a low radiation dose chest CT protocol targeting the areas of known radiographic disease to reduce radiation exposure. 
TABLE 1 Highlights of expert recommendations beyond CFF/ECFS guidelines

\begin{tabular}{|c|c|}
\hline CFF/ECSF NTM guidelines recommendations ${ }^{3}$ & Expert considerations beyond the guidelines \\
\hline $\begin{array}{l}\text { Stop chronic azithromycin once NTM is identified to avoid emergence of } \\
\text { macrolide resistance. }\end{array}$ & $\begin{array}{l}\text { Stop inhaled tobramycin to reduce risk of aminoglycoside resistance } \\
\text { and minimize use of other oral antibiotics with activity against NTM. }\end{array}$ \\
\hline $\begin{array}{l}\text { Sputum, induced sputum, bronchial washings, or bronchoalveolar lavage } \\
\text { samples can be used to test for NTM. }\end{array}$ & $\begin{array}{l}\text { Induced sputum is the } 1 \text { st preferred alternative to expectorated } \\
\text { sputum. OP swab samples plated on Burkholderia cepacia selective agar } \\
\text { can also be used to recover M. abscessus complex. }\end{array}$ \\
\hline $\begin{array}{l}\text { All aspects of CF care should be reviewed and optimized to determine } \\
\text { the clinical significance of NTM in the sputum. }\end{array}$ & $\begin{array}{l}\text { If applicable, assess response to new CFTR modulator therapy before } \\
\text { diagnosing NTM pulmonary disease and initiating NTM treatment. }\end{array}$ \\
\hline $\begin{array}{l}\text { Treatment of } M \text {. abscessus complex pulmonary disease should involve an } \\
\text { intensive phase followed by a continuation phase, and MAC pulmonary } \\
\text { disease should be treated with a daily oral antibiotic regimen } \\
\text { containing three drugs. }\end{array}$ & $\begin{array}{l}\text { Start drugs in a staggered fashion (eg, several days or a week apart) to } \\
\text { help to monitor for drug tolerance and side effects. }\end{array}$ \\
\hline $\begin{array}{l}\text { Use of therapeutic drug monitoring should be considered for individuals } \\
\text { failing to improve despite taking recommended drug regimens or for } \\
\text { those on concomitant medications with significant interactions with } \\
\text { NTM drugs. }\end{array}$ & $\begin{array}{l}\text { If a patient fails to convert to negative AFB cultures after } 6 \text { months of } \\
\text { treatment, test drug levels to ensure therapeutic dosing, check drug } \\
\text { sensitivity testing, and consider intensification of the NTM treatment } \\
\text { regimen. }\end{array}$ \\
\hline $\begin{array}{l}\text { Individuals with CF receiving NTM treatment should have expectorated } \\
\text { or induced sputum samples sent for NTM culture every 4-8 wk } \\
\text { throughout the entire course of treatment to assess the microbiological } \\
\text { response. }\end{array}$ & $\begin{array}{l}\text { For nonexpectorating patients, a bronchial wash or BAL could be timed } \\
\text { first after 3-6 mo of treatment to test for negative culture conversion, } \\
\text { and again at the end of the treatment course. Alternatively, one may } \\
\text { rely on clinical and/or radiographic outcomes to monitor treatment } \\
\text { response. }\end{array}$ \\
\hline
\end{tabular}

Abbreviations: CFF, Cystic Fibrosis Foundation; ECFS, European Cystic Fibrosis Society; NTM, nontuberculous mycobacteria; OP, oropharyngeal.

\subsection{What if the patient does not convert to negative cultures?}

In the difficult case where all treatment strategies have been exhausted and a patient fails to consistently have negative cultures, other goals of care should be considered based on a shared decision-making discussion with the patient. The panel discussed the potential role of chronic, suppressive NTM treatment. ${ }^{3}$ This could take the form of a limited NTM treatment regimen (eg, a twodrug regimen), but the panel strongly recommended avoiding monotherapy. Alternatively, one could consider scheduling planned NTM treatment periods or consider intermittent NTM treatment during periods of pulmonary exacerbation or clinical decline, with periods off of NTM treatment in between. Alternatively, one may decide to stop NTM treatment and monitor closely for clinical deterioration, in which case, reinitiating NTM treatment may be warranted.

\section{4 | CARE AFTER NTM TREATMENT}

\subsection{How should patients be managed after NTM treatment?}

Finally, the panel discussed clinical challenges that arise when caring for patients after completion of NTM treatment. For patients who have completed NTM treatment and have achieved negative AFB cultures, AFB cultures should be monitored at quarterly CF clinic visits. A common question that arises is when one could consider restarting three times weekly azithromycin for patients with chronic Pseudomonas aeruginosa infection or with frequent pulmonary exacerbations. The panel, in general, preferred to not restart chronic azithromycin in patients who have completed NTM treatment, as risk of a subsequent NTM infection ranges from $24 \%$ to $36 \%$ within 5 years. $^{2}$ Restarting chronic azithromycin could be considered for select patients following a year off treatment when the benefits of azithromycin outweigh the risk of macrolide resistance if a subsequent NTM infection occurred, a decision best made through a shared decision-making process with the patient and physician (Table 1).

In conclusion, clinical challenges and areas of uncertainty exist throughout all phases of managing pulmonary NTM infections in people with CF. Important through all phases is a well-organized and closely monitored management plan that is mutually agreed upon by the provider, CF care team, and patient.

\section{ACKNOWLEDGMENTS}

This manuscript was funded by grants from the Cystic Fibrosis Foundation to LJC (CAVERL17A0), SLM (MARTIN17K0); National Institutes of Health to LJC (K23HL136934).

\section{CONFLICT OF INTERESTS}

SLM: Advisory board for Paratek Pharmaceuticals. SHK: Speaker and the advisory board for Insmed. CSH: Speaker fees and consultancy work for Insmed. 


\section{ORCID}

Stacey L. Martiniano (D) http://orcid.org/0000-0002-9042-3642

Charles R. Esther (D) http://orcid.org/0000-0002-8081-2986

Edith T. Zemanick (D) http://orcid.org/0000-0002-7507-9337

Lindsay J. Caverly (D) http://orcid.org/0000-0001-8658-0867

\section{REFERENCES}

1. Cystic Fibrosis Foundation. Patient Registry Annual Data Report. 2017.

2. Martiniano SL, Sontag MK, Daley CL, Nick JA, Sagel SD. Clinical significance of a first positive nontuberculous mycobacteria culture in cystic fibrosis. Ann Am Thorac Soc. 2014;11:36-44.

3. Floto RA, Olivier KN, Saiman L, et al. US cystic fibrosis foundation and European Cystic Fibrosis Society consensus recommendations for the management of nontuberculous mycobacteria in individuals with cystic fibrosis. Thorax. 2016;71:i1-i22.

4. Griffith DE, Aksamit T, Brown-Elliott BA, et al. An official ATS/IDSA statement: diagnosis, treatment, and prevention of nontuberculous mycobacterial diseases. Am J Respir Crit Care Med. 2007;175: 367-416.

5. Hoppe JE, Towler E, Wagner BD, Accurso FJ, Sagel SD, Zemanick ET. Sputum induction improves detection of pathogens in children with cystic fibrosis. Pediatr Pulmonol. 2015;50:638-646.
6. Ronchetti K, Tame JD, Paisey C, et al. The CF-Sputum Induction Trial (CF-SpIT) to assess lower airway bacterial sampling in young children with cystic fibrosis: a prospective internally controlled interventional trial. Lancet Respir Med. 2018;6:461-471.

7. Esther CR, Kerr A, Gilligan PH. Detection of Mycobacterium abscessus from deep pharyngeal swabs in cystic fibrosis. Infect Control Hosp Epidemiol. 2015;36:618-619.

8. Olivier KN, Weber DJ, Lee JH, et al. Nontuberculous mycobacteria. II: nested-cohort study of impact on cystic fibrosis lung disease. Am J Respir Crit Care Med. 2003;167:835-840.

9. Guimbellot JS, Acosta EP, Rowe SM. Sensitivity of ivacaftor to drugdrug interactions with rifampin, a cytochrome P450 3A4 inducer. Pediatr Pulmonol. 2018;53(5):E6-E8.

10. Gilljam M, Berning SE, Peloquin CA, Strandvik B, Larsson LO. Therapeutic drug monitoring in patients with cystic fibrosis and mycobacterial disease. Eur Respir J. 1999;14(2):347-351.

How to cite this article: Martiniano SL, Esther CR, Haworth CS, Kasperbauer SH, Zemanick ET, Caverly LJ. Challenging scenarios in nontuberculous mycobacterial infection in cystic fibrosis. Pediatric Pulmonology. 2020;55:521-525.

https://doi.org/10.1002/ppul.24604 